\title{
Thermoluminescence color images from quartzs of beach sands
}

\author{
Tetsuo Hashimoto, AKIRA Koyanagi, KYOICHI YoKosaKa, \\ YASUO HAYASHI and TAKESHI SOTOBAYASHI \\ Department of Chemistry, Faculty of Science, Niigata University \\ Ikarashi-ninocho, Niigata 950-21, Japan
}

(Received August 6, 1985: Accepted January 29, 1986)

\begin{abstract}
Some quartzose beach sands, collected from the northern part of Niigata district, were examined by thermoluminescence color image (TLCI) analyses. Analyses were performed after purification of the quartz fraction by magnetic separation. Purified samples underwent chemical etching followed by gamma-ray irradiation. The proportion of red TL-emitting grains to blue ones seems to be related to the origin of the respective beach sands. Red TL grains are tentatively attributable to a volcanic dominated source, whereas the blue ones are mainly weathering products of plutonic rocks such as granite. TL-spectrophotometric analyses have been attempted to obtain more quantitative information on the TL-emission of the two different colors for these beach sands. The intensity ratios of red to blue TL were evaluated from analyses of respective glow-curves, corresponding to different wavelength regions. The ratios were concordant with the TLCI parterns. The applications of TLCI and TL-spectrophotometry to geological and archaeological samples are also suggested.
\end{abstract}

\section{INTRODUCTION}

When high energy radiation passes through an insulating material, its constituent atoms are excited and ionized. Some of the excited electrons cannot return to their original atomic sites and a small fraction of them are trapped in lattice defects or by impurity atoms in the matrix. These trapped electrons can be reexcited and diffuse within the insulating material by heating. Some of these electrons may combine with positive holes accompanied by light emission known as thermoluminescence (abbreviated to TL hereafter). In the case of geological materials, natural TL intensity can be utilized as an absolute dating technique (McDougall, 1968; Aitken, 1974).

In the course of previous TL investigation of geological materials, the authors noticed that TL from some sliced granites (Hashimoto et al., 1981, 1983) can be colorphotographed using a conventional photographic system. The photographic TL color image (abbreviated to TLCI) was found to allow visual separation between red and blue TL-emitting quartz grains (Hashimoto et al., 1986a).

This TLCI method was applied to the quartz grains separated from beach sands. The TL glow-curve analyses, combined with spectrometric measurements, can make a valuable contribution to our knowledge of the formational history of beach sands.

\section{EXPERIMENTAL}

\section{Sample preparation for TLCI}

Several beach sand samples were collected from the coast in the north of Niigata district as shown in Fig. 1. The sand samples were thoroughly washed, to remove clayey substances and adhered salts, with deionized water and dried in an oven maintained at $60^{\circ} \mathrm{C}$. After sieving, +32 mesh fractions were collected and further purified with a Frantz isodynamic separator with a front slope of $25^{\circ}$ and a side slope of $15^{\circ}$ at $1.7 \mathrm{~A}$. The non-magnetic fractions were etched with both $6 \mathrm{M} \mathrm{NaOH}$ and $6 \mathrm{M}$ $\mathrm{HCl}$ each for 10 minutes then with $47 \% \mathrm{HF}$ 


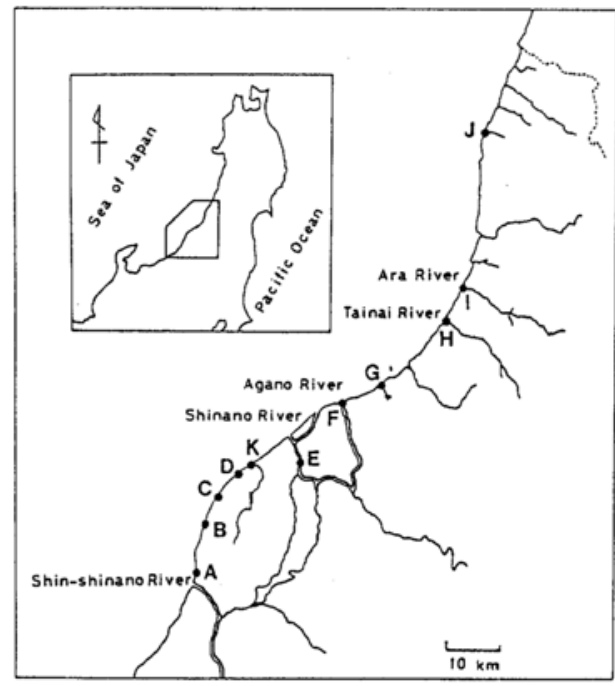

Fig. 1. Sampling locations of beach sands. A: Nozumi Beach, B; Maze Beach, C: Kakumi Beach, D: Kakuda Beach, E: Shinano River, F: Agano River, G: Higashi Port, H: Tainai River, I: Ara River, J: Ima River, K: Ikarashi Beach.

solution for one hour at $25^{\circ} \mathrm{C}$ under ultrasonic agitation. With this method, concentrated quartz sand fractions were obtained after further purification using 62 mesh sieving treatment to remove small zircon grains. In some cases the quartz grains were carefully hand-picked under a stereoscopic microscope.

\section{Sample irradiation and TL-observations}

A Co-60 gamma-ray source in the Research Reactor Institute, Kyoto University, was used as a standard radiation field. Alternatively, an $\mathrm{X}$-ray irradiation was applied using a fluorescent X-ray analysis apparatus (Geiger Flex SX3063P, Rigakudenki Co. Ltd.). By comparison between the Co-60 radiation field and X-ray irradiation position, the absorbed dose of the latter facility was evaluated at $9.42 \mathrm{kGy}$ for 3 min-irradiation.

The TLCI photographic method has been described in detail in previous papers (Hashimoto et al., 1983, 1986a). Sakura-400 (ASA) color films and quartz samples of about $10 \mathrm{mg}$ each were used in the experiments.

\section{Measurement of TL-spectrum}

TL-spectra were measured by using a micro-

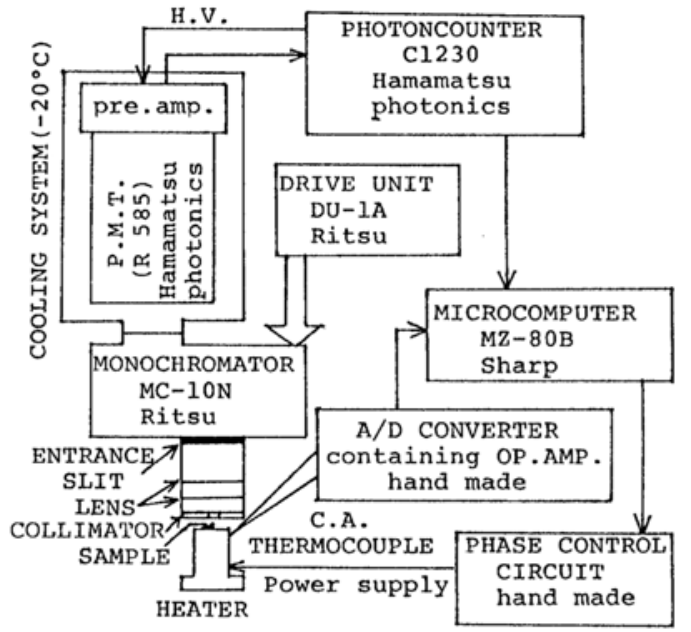

Fig. 2. Block diagram of TL-spectrum measurement assembly.

computer-assisted photon-counting system as described in a previous paper (Hashimoto et al., 1986b). A monochromator (MC-10N, Ritsu) coupled with a drive unit (Model DU-1 A, Ritsu) was installed between a heater device and the photomultiplier tube as illustrated in Fig. 2. The monochromator was equipped with a diffraction grating of 1,200 grooves $/ \mathrm{mm}$ and weak TL-light was effectively collected by two focal lenses. The TL from 5-10mg quartz samples was measured over a wavelength region of 200 to $700 \mathrm{~nm}$ with this system.

\section{Results AND Discussion}

Some typical thermoluminescence color images (TLCI) of irradiated quartz grains are presented in Fig. 3. This figure shows many types of differently colored TL emissions (such as, red, pink, violet, blue and yellow). The dominant emissions from the quartz grains, however, are red and blue as described already (Hashimoto et al., 1986b, c). In that paper, the authors tentatively concluded, on the basis of the instrumental neutron activation analysis, that the emissions should depend on the amounts of impurity atoms, especially light rare earth elements (probably Sm or Eu). There was no difference between the crystallographic pro- 
perties of quartz grains emitting the differently colored TL. These colors were verified as significantly dependent on the origin of respective quartz grains. Blue ones are derived from the quartz in plutonic rocks. A relatively slow solidification of the rocks was sufficient to expel impurity elements from quartz. However, red ones are mainly due to quartz with higher impurity contents. These were formed under relatively rapid cooling conditions like in a volcanic ash layer.

From this viewpoint, Fig. 3 implies some interesting information relating to the origin of respective beach sands and the intrinsic proportions of each color. In the southern district, three beach sands of Nozumi, Maze, and Kakuda beaches are identified as clearly having been supplied from the Shinano River, the longest river in Japan, from aspects of ground configuration and heavy mineral content. From their TLCI patterns these three sands have similar proportions of red and blue TLCI. This is consistent with their supply from the Shinano River, which flows through complexly mixed areas consisting of volcanic and plutonic rocks or their weathered equivalents. Kakumi sample gives the strongest red TL among the samples. Although the appearance of original sands suggests that all three sands originate from the Shinano River, Kakumi Beach is located in a small inlet surrounded by volcanic mountains and has been closed for a long period to the supply of sands derived from other areas (Shirai et al., 1976). This sand sample seems to contain mainly native quartz sands which originated from volcanic ashes. High transparent quartz sand content of Kakumi quartz sands has been shown to correspond to a volcanic quartz origin in preceding TLCI experiments (Hashimoto et al., 1986a). There is an apparent difference between Agano (and Higashi Port) and Shinano River samples; Agano River and Higashi Port sands have an abundant red fraction relative to blue TL grains. This could also imply that the Agano River drains a volcanic dominated source. In fact, the Agano River does carry much volcanically derived quartzs owing to the eruption of Numazawa volcano in approximately 5,000 Y B.P. (Niigata Ancient Dune Research Group, 1978). On the other hand, the TLCIs from three remaining samples, Tainai, Ara, and Ima rivers, seldom contain the red fraction. This suggests that the north district of Niigata Prefecture which consists mainly of granitic rock bodies (Asahi mountainous area, with an age range of $60-200 \mathrm{Ma}$ ) as the sources of blue TL-emitting quartz sands.

Although these TLCI-patterns may serve to trace qualitatively the origin of quartz, further information should be extracted only from quantitative spectral measurements. From this standpoint, TL-spectrophotometric analyses have been attempted using the spectrophotometric system illustrated in Fig. 2. In the course of the TL-spectrophotometry, two difficulties are inevitably encountered; one is sensitivity calibration of the present measuring system over $200-700 \mathrm{~nm}$ range, and the other is changes of TL-intensity with very weak luminescence or heating. To overcome the former, a standard light source (JC-24V, Ushio) was employed and detection efficiency was converted into a relative correction factor which was normalized to unity at $400 \mathrm{~nm}$ as represented in Fig. 4 (A). The quartz sands from Kakumi Beach were taken as a representative sample showing strong red and weak blue TL-emission, as seen in Fig. 3, and were spectrophotometrically analyzed. The observed spectra without and with the correction are shown in Fig. 4 (A) and (B), respectively. From the corrected spectrum (B), it was confirmed that this TL-spectrum consists of a blue spectrum part having a peak at around $470 \mathrm{~nm}$ and red part having a peak at around $600 \mathrm{~nm}$. It was found, however, that poorer detection efficiency for longer wavelengths results in a weak peak. This longer wavelength region corresponds to the red color region, so that the spectrum areas belonging to each spectrum region are found to be unsuitable for quantitative evaluation of the intensity ratio of red emission to blue one. Another correction based on the change of TL-intensity should be considered for quantitative discussion. These 

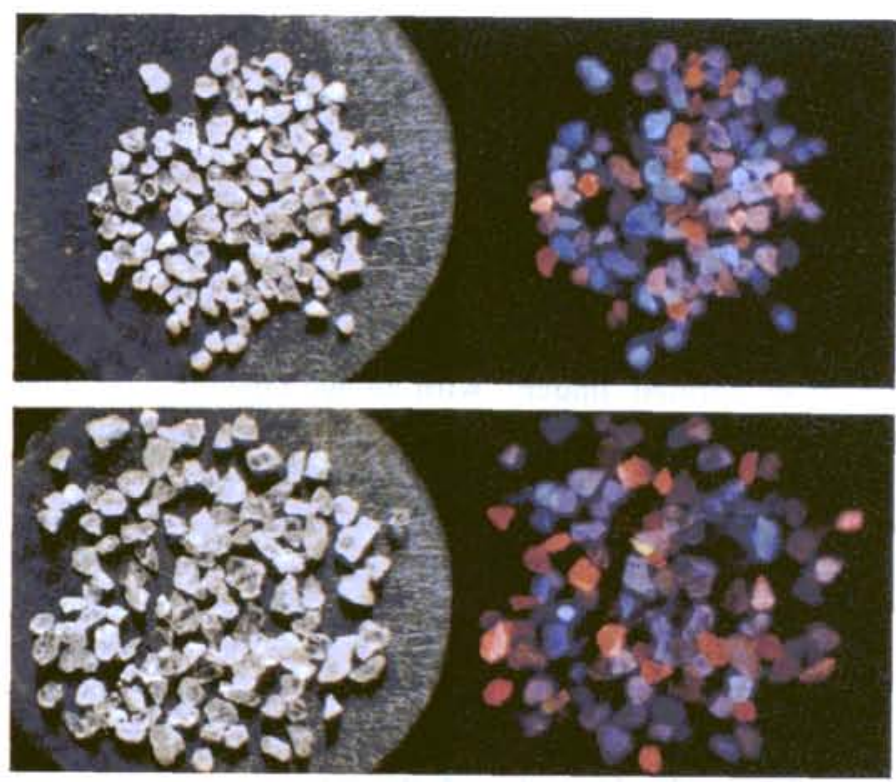

으
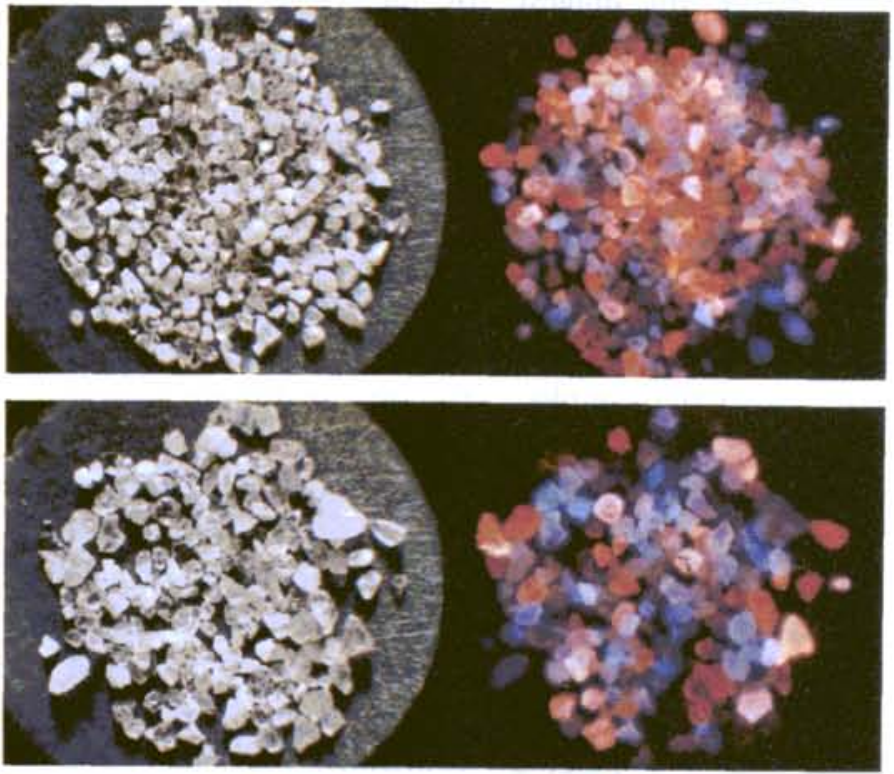

봉

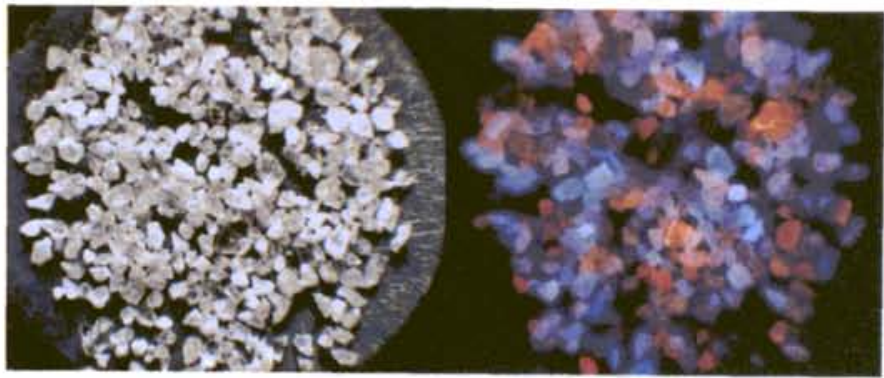



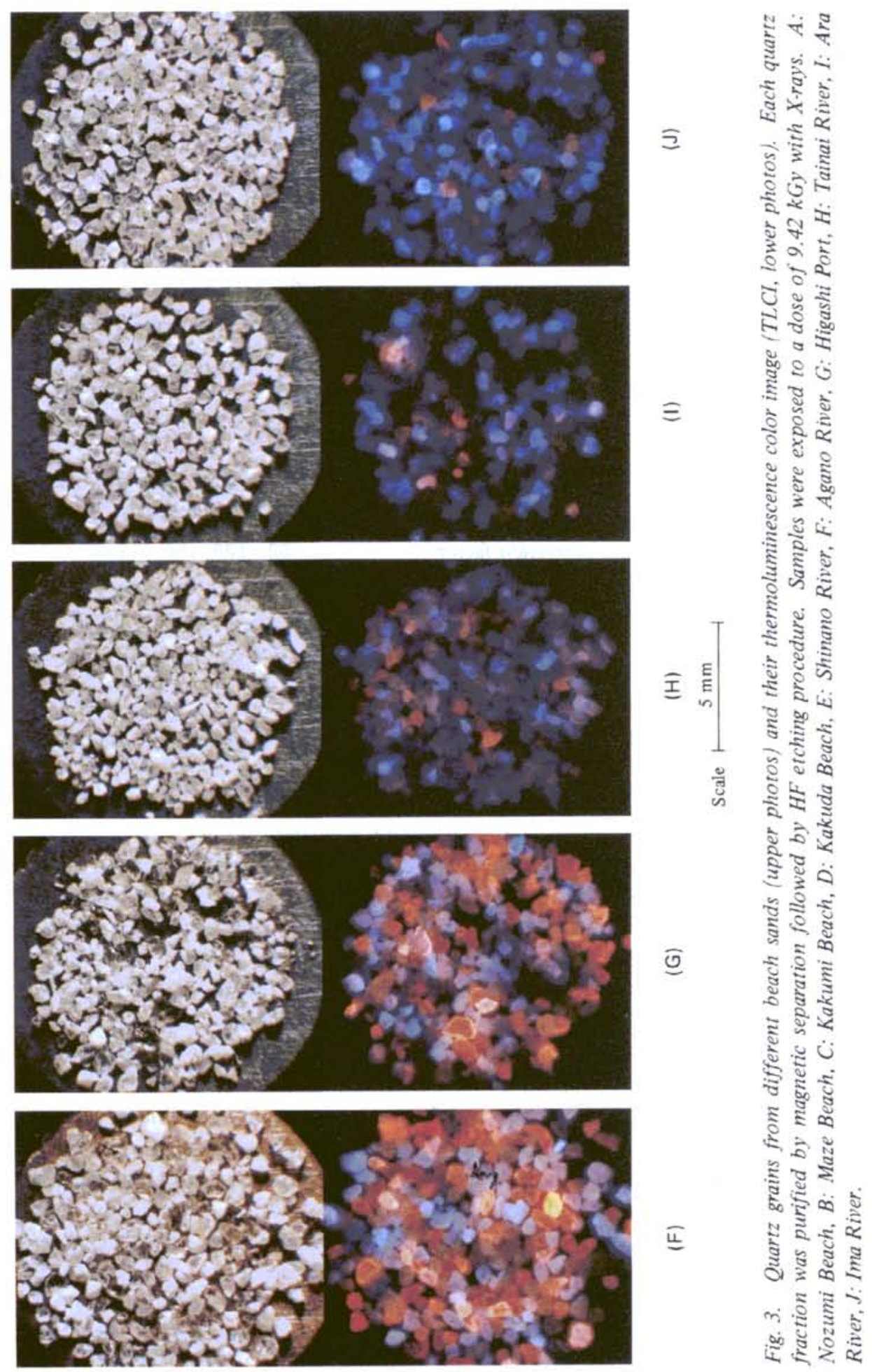

$\therefore \rightarrow$

站的

造高产

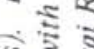

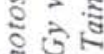

a

원

วิ

$2 \div 2$

पi

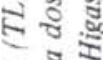

品 2

$\left.c^{2}+x^{2}+2\right)$

C. $2,2 \times$

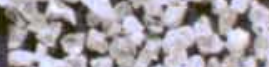

ations

Extinas

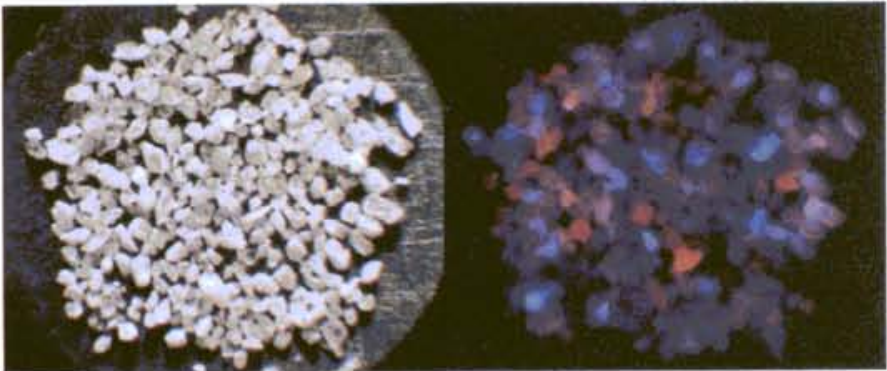

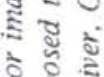

8 2

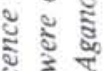

造

ํํำ

है

บั บั

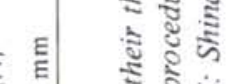

舟事

ริ

บะ

空资

के

要的

ปั

ปิ)

ล है है

$\because \ddot{u}$

ษั

E

$\rightarrow$ บั

政

$\infty$ 实舟

ปิ

m. ฐี

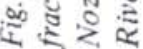




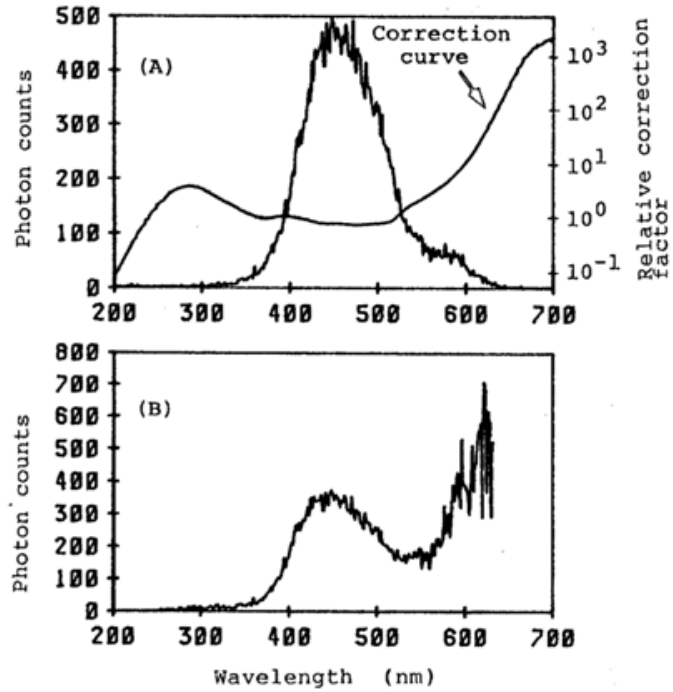

Fig. 4. A typical TL-spectrum (A) and its corrected one (B) obtained by applying the relative correction factor of the measurement assembly. The sample used, collected from Kakumi Beach, was exposed to a dose of $9.32 k G y$ with $\mathrm{Co}-60$ source.

corrections are troublesome as they bring about more unreliable results owing inherent uncertainties.

For these reasons, the following procedures were adopted; two wavelength regions, 570-630 and $440-500 \mathrm{~nm}$, were selected for measurements of red and blue TL-quantities, respectively. Two separate runs of TL glow-curves were measured over respective wavelength regions and typical results from Kakumi Beach sands are illustrated in Fig. 5. The red luminescence (A) tends to give a higher temperature peak although the blue one certainly gives a stronger peak on its lower temperature side (approximately $190^{\circ} \mathrm{C}$ ) and a broad peak in the higher temperature region (B). Similar glowcurve tendencies were initially found in quartz contained in a volcanic ash (SK-020, Uonuma standard strata) and a pegmatite quartz rock (Ishikawa-Machi, Fukushima Prefecture) used as representative material giving red and blue TLemission, respectively (Hashimoto et al., 1986a, c). The total amount of TL-light was evaluated for each sample from this glow-curve and the relative correction factor as indicated in Fig. 4
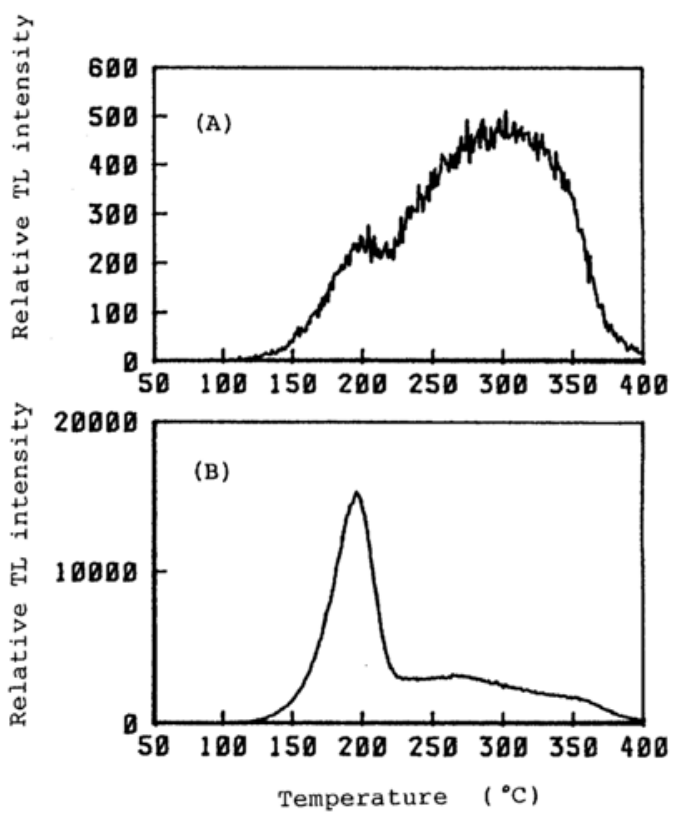

Fig. 5. Typical glow-curves when the measuring wavelength regions were fixed to $570-630 \mathrm{~nm}(A)$ and $440-500 \mathrm{~nm}$ (B) as representative red and blue $T L$ spectrum regions, respectively. The quartz sample was the same as that used in Fig. 4. The heating rate was $0.1^{\circ} \mathrm{C} / \mathrm{s}$.

(A) was determined. The present data treatments were initially checked with regard to the reproducibility of the final values. The Ikarashi Beach sands were collected from three different points spaced about $500 \mathrm{~m}$ apart from each other. The results agree fairly well with each other and within the bounds of experimental error. On this basis, five beach sands were used for glow-curve analyses. The results obtained are summarized in Table 1. In this table, total luminescence value was evaluated by integrating the differential photon numbers as seen in the red and blue TL glow-curves (cf. Fig. 5). The higher temperature region ranging from 240 to $400^{\circ} \mathrm{C}$ was adopted to avoid TL-decay effects of the lower temperature region relative to the higher temperature side. In the second and third columns, the highest sensitivity of red TL is assigned to the Agano River sand sample whereas the same high sensitivity of blue TL appears in the Ikarashi Beach sands. This evidently 
Table 1. Ratios of red to blue TL intensities obtained from TL-glow curves

\begin{tabular}{llccc}
\hline Sand sample & $\begin{array}{c}\text { Red } \\
\text { luminescence (R) }\end{array}$ & $\begin{array}{c}\text { Blue } \\
\text { luminescence (B) }\end{array}$ & R/B ratios & $\begin{array}{c}\text { corrected } \\
\text { R/B ratios }\end{array}$ \\
\hline \hline Kakumi Beach & $4.68( \pm 0.41) \times 10^{4}$ & $4.09( \pm 0.29) \times 10^{5}$ & $1.15( \pm 0.13) \times 10^{-1}$ & $1.82 \pm 0.21$ \\
Agano River & $6.64( \pm 0.71) \times 10^{4}$ & $1.29( \pm 0.26) \times 10^{6}$ & $5.16( \pm 1.18) \times 10^{-2}$ & $0.82 \pm 0.19$ \\
Ikarashi Beach & $5.04( \pm 0.56) \times 10^{4}$ & $1.49( \pm 0.05) \times 10^{6}$ & $3.38( \pm 0.39) \times 10^{-2}$ & $0.54 \pm 0.06$ \\
Ara River & $7.09( \pm 1.17) \times 10^{3}$ & $4.87( \pm 0.88) \times 10^{5}$ & $1.46( \pm 0.36) \times 10^{-2}$ & $0.23 \pm 0.06$ \\
Ima River & $5.89( \pm 0.90) \times 10^{3}$ & $3.24( \pm 0.49) \times 10^{5}$ & $1.82 \pm 0.33) \times 10^{-2}$ & $0.29 \pm 0.05$ \\
\hline
\end{tabular}

Quartz sand samples each $5 \mathrm{mg}$ were subjected to the measurements of glow-curves twice after exposure to a dose of 9.32kGy from a Co-60 source. Two wavelength regions, 570-630 and 440-500 nm, were used for the measurement of red and blue TL-glow curves, respectively. The final luminescence values were evaluated by integrating photon numbers from each glow-curve over the temperature range of 240 to $400^{\circ} \mathrm{C}$. $R$ and $B$ represent the reduced photon numbers for $1 \mathrm{mg}$ sample weight and errors estimated from photoncounting statistics. The corrected $R / B$ ratios were computed by applying the relative correction factor.

supports the hypothesis of different origins for red and blue TL-emitting quartz minerals, although apparent sensitivity differences within one order of magnitude are observed among TL-materials of the same color. In general higher TL-sensitive minerals contain many impurities and lattice defects within their crystals, reflecting a relatively rapid rate of crystalization from magma. Using intensity ratios of red to blue TL and a related correction factor, we present corrected ratios of red to blue $\mathrm{TL}$ in the last column in descending order beginning with stronger red TL-emission sand samples. This order may be justified from visual observation TLCI patterns in Fig. 3; the highest ratio is for the Kakumi Beach sands, whereas the lowest one is from the Ara River sands. Thus, it should be emphasized that TLcoloration from small amounts of quartz sand (5-10 mg) should offer us useful information which has been unavailable to other scientific investigation techniques. Although the basic spectrometric nature of TL has been partially clarified in the present experiments, the distribution of rare earth elements, responsible for changes of TL-color, yields knowledge related to geological conditions for distribution of rare earth elements among minerals including quartz, formation and/or metamorphic temperature, pressure, and oxygen fugacity (Masuda, 1962; Henderson, 1984).

In future studies, the present treatments of TL phenomena should be applicable to a variety of geoscientific or related fields since quartz grains exist commonly in many geological samples and are resistant to weathering. As no change of coloration has been confirmed even after heating the quartz up to $1,200^{\circ} \mathrm{C}$, the origin of artificial goods including archaeological pottery could be searched by TLCI observation with glow-curve analyses of constituent quartz grains. In addition, quartz minerals themselves store naturally occurring trapped electrons in lattice defects or impurity atoms. In the near future, extremely weak TLCI patterns due to de-excitation of naturally trapped electrons will be effectively utilized with the aid of imageintensifier spectrophotometry as proposed by Walton (1982). Such use of natural TL is recommendable as there is no need to use gamma or X-ray irradiation.

\section{Conclusion}

The origin of beach sands were clarified by using TLCI patterns of gamma-irradiated quartz grains. Distinct TL-coloration of quartz sands appear to be distinguishable into two main parts, i.e. red and blue ones. The intensity ratio of these colors evidently reflects the different origins of beach sands collected from different districts. In advanced analyses using the TLspectrum, more quantitative knowledge has been used to examine the origin of quartz sands, on the basis of red TL-quartz derivation from volcanic ash layers and blue TL-quartz derived 
from plutonically formed rocks (including their weathering products) (Hashimoto et al., 1986a, c). These newly developed techniques using TL are useful not only in geological investigation, but in those of applied sciences, including archaeology, oceanology and meteorology.

Acknowledgement-The authors would like to thank Messers. T. Warashina and R. Matsushita of the Research Reactor Institute, Kyoto University, for their kind help with Co-60 gamma-ray irradiation and valuable technical assistance. They are also indebted to Dr. J. Aitchison, University of Otago, New Zealand, for reading the manuscript and making valuable suggestion.

The present work was supported in part by Grant-in Aids for Fundamental Scientific Research from the Ministry of Education and from the Japan Association of Chemistry.

\section{REFER ENCES}

Aitken M. J. (1974) Physics and Archaelogy. Clarendon Press, Oxford.

Hashimoto T., Hayashi Y., Koyanagi A., Yokosaka K. and Kimura K. (1986a) Red and blue colouration of thermoluminescence from natural quartz sands. Nucl. Tracks Radiat. Meas. in press.

Hashimoto T., Kimura K., Koyanagi A., Takahashi K. and Sotobayashi T. (1983) New colour-photo- graphic observation of thermoluminescence from sliced rock samples. Radioisotopes (Tokyo) 32, 525-53 (Japanese).

Hashimoto T., Koyanagi A., Takahashi K., Kimura K. and Hayashi Y. (1986b) A computer controlled thermoluminescence reader with a ceramic heating plate. J. Radioanal. Nucl. Chem., 102, 455-464.

Hashimoto T., Takahashi K. and Sotobayashi T. (1981) Observation of thermoluminescence from natural minerals. 8th Annu. Meet. Radioisot. Phys. Sci. Ind. 30a-II-5. 89 (Japanese).

Hashimoto T., Yokosaka K. and Habuki H. (1986c) Emission properties of thermoluminescence from quartzs - blue and red TL-response on adsorbed dose. Submitted to Nucl. Tracks Radiat. Meas.

Henderson P. (ed.) (1984) Rare Earth Element Geochemistry. Elsevier.

Masuda A. (1962) Regularities in variation of relative abundances of lanthanide elements and an attempt to analyse separation-index patterns of some minerals. J. Earth Sci., Nagoya Univ. 10, 173-187.

Niigata Ancient Dune Research Group (1978) The sand of Niigata sand dunes. II. Daiyonki Kenkyu 17, 25-36 (Japanese).

Shirai T., Tsuda K., Chihara K. and Hasegawa Y. (1976) Geology of the Yahiko-Kakuda mountainland, Niigata Prefecture. Contrib. Dept. Geol. Mineral., Niigata Univ 4, 111-118 (Japanese).

Walton A. J. (1982) An image-intensifier spectrograph for thermoluminescence studies. PACT 6, 524-534. 